\title{
Structural Model and Diagram of Multilayer Electro Magneto Elastic Actuator for Nanotechnology
}

\author{
Afonin $\mathrm{SM}^{*}$ \\ National Research University of Electronic Technology, MIET, Moscow, Russia
}

*Corresponding author: Afonin SM, National Research University of Electronic Technology, MIET, Moscow, Russia.

Received Date: November 10, 2019

Published Date: November 18, 2019

\begin{abstract}
We obtained the structural model and the structural diagram of the multilayer electro magneto elastic actuator for nanotechnology in contrast to electrical equivalent circuits of the piezo transmitter and piezo receiver, the vibration piezo motor. We determined the structural model, the structural diagram and the matrix transfer function of the multilayer electro magnetoelastic actuator for nanotechnology.
\end{abstract}

Keywords: Structural model; Structural diagram; Multilayer electro magneto elastic actuator; Multilayer piezo actuator; Matrix transfer function

\section{Introduction}

The multilayer electro magneto elastic actuator nano and micro displacement with the piezoelectric, piezomagnetic, electrostriction, magnetostriction effects is used in the control system for nanotechnology in scanning tunneling microscopy, atomic force microscopy and adaptive optics in the range of movement from few nanometers to tens of micrometers [1-32]. We obtained the structural model and the structural diagram of the multilayer electro magneto elastic actuator in contrast to electrical equivalent circuits of the piezo transducer, the vibration piezo motor [1-11]. We used the equation of the electro magneto elasticity, the equivalent mechanical quadrupole and the boundary conditions of the multilayer actuator for the structural model and the structural diagram of the multilayer actuator [8-28]. We received the matrix transfer function of the multilayer electro magneto elastic actuator from its structural model.

\section{Structural Model and Diagram}

We determined the structural model and the structural diagram of the multilayer electro magneto elastic actuator for nanotechnology and nanomedicine in difference from Cady and Mason electrical equivalent circuits of the piezo transducer. We used the method of the mathematical physics with Laplace transform for the determination the structural model and the structural diagram of the multilayer electro magneto elastic actuator for nanotechnology and nanomedicine $[8,14,32]$.
The equation $[8,9,11,25,26]$ of the electro magneto elasticity has the following form

$$
S_{i}=v_{m i} \Psi_{m}+s_{i j}^{\Psi} T_{j}
$$

Where $S_{i}$ is the relative displacement, $v_{m i}$ is the coefficient of electro magneto elasticity: $d_{m i}$ piezo module or magneto strictive coefficient, $\Psi_{m}$ is control parameter in the form of electric $E_{m}$ , magnetic $H_{m}$ field strengths or electric $D_{m}$ induction, $s_{i j}^{\psi}$ is the elastic compliance with $\Psi=$ const,$T_{j}$ is the mechanical stress, $i, j$, $m$ are the indexes.

We received for the multilayer electro magneto elastic actuator the causes force in the form

$$
F=v_{m i} S_{0} \Psi_{m} / s_{i j}^{\Psi}
$$

where $S_{0}$ is the cross sectional area of the actuator.

We have matrix the equivalent mechanical quadrupole of the multilayer electro magneto elastic actuator [25] in the following form

$$
[M]^{n}=\left[\begin{array}{cc}
\operatorname{ch}(l \gamma) & Z_{0} \operatorname{sh}(l \gamma) \\
\frac{\operatorname{sh}(l \gamma)}{Z_{0}} & \operatorname{ch}(l \gamma)
\end{array}\right]
$$

where $l$ is length of the multilayer actuator for the longitudinal piezo effect $l=n \delta$, for the transverse piezo effect, for the shift piezo 
effect $l=n b$, and $\delta, h, b$ are the thickness, the height, the width for the piezo layer.

We obtained the system of the equations for the structural model and the structural diagram of the multilayer electro magneto elastic actuator on Figure 1. We have the structural model of the multilayer electro magneto elastic actuator in result analysis the equation of the force that causes deformation, the equivalent mechanical quadrupole and the boundary conditions equations with the forces on faces of the actuator in the following form

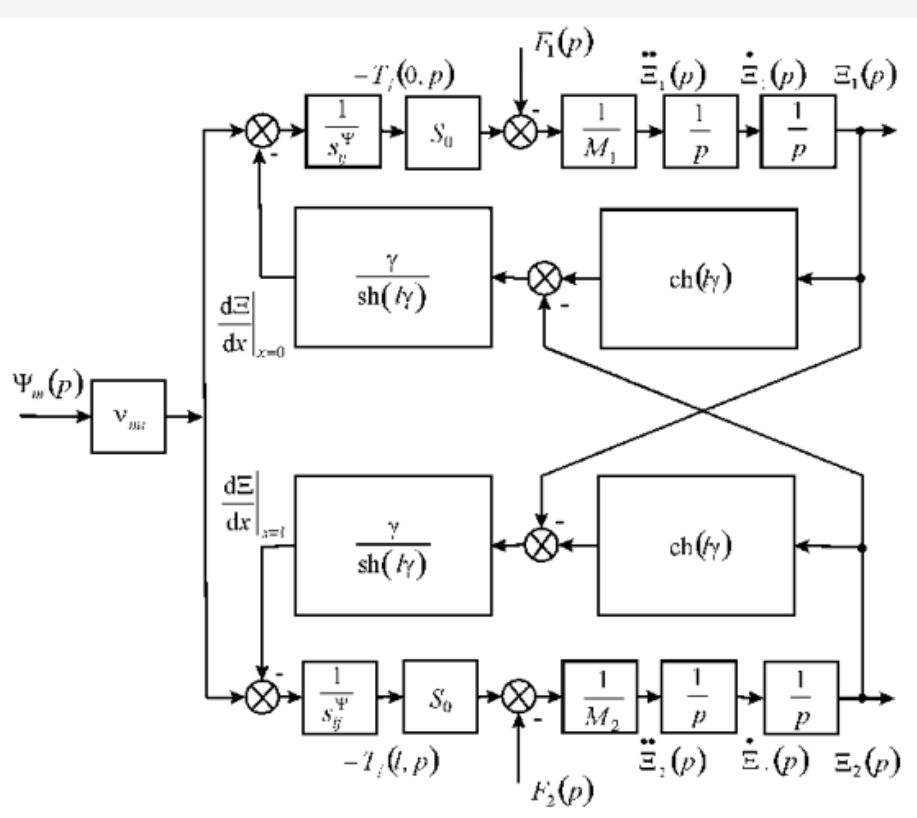

Figure 1: Structural diagram of multilayer electro magneto elastic actuator.

$\Xi_{1}(p)=\left[1 /\left(M_{1} p^{2}\right)\right] \times$

$\times\left\{-F_{1}(p)+\left(1 / \chi_{i j}^{\Psi}\right)\left[v_{m i} \Psi_{m}(p)-[\gamma / \operatorname{sh}(l \gamma)]\left[\operatorname{ch}(l \gamma) \Xi_{1}(p)-\Xi_{2}(p)\right]\right]\right\}$

$\Xi_{2}(p)=\left[1 /\left(M_{2} p^{2}\right)\right] \times$

$\times\left\{-F_{2}(p)+\left(1 / \chi_{i j}^{\Psi}\right)\left[v_{m i} \Psi_{m}(p)-[\gamma / \operatorname{sh}(l \gamma)]\left[\operatorname{ch}(l \gamma) \Xi_{2}(p)-\Xi_{1}(p)\right]\right]\right\}$

where $v_{m i}=\left\{\begin{array}{l}d_{33}, d_{31}, d_{15} \\ g_{33}, g_{31}, g_{15} \\ d_{33}, d_{31}, d_{15}\end{array} \Psi_{m}=\left\{\begin{array}{l}E_{3}, E_{1} \\ D_{3}, D_{1} \\ H_{3}, H_{1}\end{array}\right.\right.$,

$$
s_{i j}^{\Psi}=\left\{\begin{array}{l}
s_{33}^{E}, s_{11}^{E}, s_{55}^{E} \\
s_{33}^{D}, s_{11}^{D}, s_{55}^{D} \\
s_{33}^{H}, s_{11}^{H}, s_{55}^{H},
\end{array}\right.
$$$$
c^{\Psi}=\left\{\begin{array}{l}
c^{E} \\
c^{D} \\
c^{H}
\end{array} \quad \gamma=\left\{\begin{array}{l}
\gamma^{E} \\
\gamma^{D} \\
\gamma^{H}
\end{array}, \quad l=\left\{\begin{array}{l}
\delta \\
h \\
b, \chi_{i j}^{\Psi}=s_{i j}^{\Psi} / S_{0} .
\end{array}\right.\right.\right.
$$

From the structural model we have the matrix transfer function of the multilayer electro magneto elastic actuator for nanotechnology in the form

$$
[\Xi(p)]=[W(p)][P(p)]
$$

where $[\Xi(p)],[W(p)],[P(p)]$ are the matrices of the displacements the faces, the transfer functions, and the control parameters.
We have at $t \rightarrow \infty$ the static displacements the faces of the voltage-controlled multilayer piezo actuator for the longitudinal piezo effect and the inertial load for $m<<M_{1}, m<<M_{2}$, where $m$ is the mass of the multilayer piezo actuator, $M_{1}, M_{2}$ are the load masses, and the forces on faces $F_{1}(t)=F_{2}(t)=0$, in the form

$$
\begin{gathered}
\xi_{1}(\infty)=\lim _{p \rightarrow 0} p W_{11}(p)(U / \delta) / p=d_{33} n U M_{2} /\left(M_{1}+M_{2}\right) \\
\xi_{2}(\infty)=\lim _{p \rightarrow 0} p W_{21}(p)(U / \delta) / p=d_{33} n U M_{1} /\left(M_{1}+M_{2}\right) \\
\xi_{1}(\infty)+\xi_{2}(\infty)=d_{33} n U
\end{gathered}
$$

where $U$ is the voltage on the actuator.

Therefore, the static displacements the faces of the multilayer piezo actuator at $d_{33}=4 \cdot 10^{-10} \mathrm{~m} / \mathrm{V}, n=4, U=50 \mathrm{~V}, M_{1}=1 \mathrm{~kg}$ and $M_{2}=4 \mathrm{~kg}$ we obtained the static displacements of the faces the multilayer piezo actuator $\xi_{1}(\infty)=64 \mathrm{~nm}, \xi_{2}(\infty)=16 \mathrm{~nm}$, $\xi_{1}(\infty)+\xi_{2}(\infty)=80 \mathrm{~nm}$

\section{Conclusion}

We obtained the structural model and the structural diagram of the multilayer electro magneto elastic actuator for nanotechnology with the mechanical parameters in the form the displacement and the force in the difference from Cady and Mason electrical equivalent circuits of the piezo transducer.

We determined the structural model, structural diagram and the matrix transfer function of the multilayer electro magneto elastic actuator for nanotechnology. 
We received the characteristics of the multilayer electro magneto elastic actuator with using its the matrix transfer function.

\section{Acknowledgement}

None.

\section{Conflict of Interest}

No conflict of interest.

\section{References}

1. Schultz J, Ueda J, Asada H (2017) Cellular Actuators. ButterworthHeinemann Publisher, Oxford, 382 p.

2. Afonin SM (2006) Absolute stability conditions for a system controlling the deformation of an elecromagnetoelastic transducer. Doklady mathematics 74(3): 943-948.

3. Zhou S, Yao Z (2014) Design and optimization of a modal-independent linear ultrasonic motor. IEEE transaction on ultrasonics, ferroelectrics, and frequency control 61(3): 535-546.

4. Przybylski J (2015) Static and dynamic analysis of a flextensional transducer with an axial piezoelectric actuation, Engineering structures 84: $140-151$.

5. Uchino K (1997) Piezoelectric actuator and ultrasonic motors. Boston, MA: Kluwer Academic Publisher, p. 347.

6. Karpelson M, Wei GY, Wood RJ (2012) Driving high voltage piezoelectric actuators in microrobotic applications. Sensors and Actuators A: Physical 176: 78-89.

7. Afonin SM (2015) Block diagrams of a multilayer piezoelectric motor for nano- and microdisplacements based on the transverse piezoeffect. Journal of computer and systems sciences international 54(3): 424-439.

8. Afonin SM (2008) Structural parametric model of a piezoelectric nanodisplacement transduser. Doklady physics 53(3): 137-143.

9. Afonin SM (2006) Solution of the wave equation for the control of an elecromagnetoelastic transduser. Doklady mathematics 73(2): 307-313.

10. Cady WG (1946) Piezoelectricity: An introduction to the theory and applications of electromechancial phenomena in crystals. McGraw-Hill Book Company, New York, London, 806 p.

11. Mason W (1964) Physical Acoustics: Principles and Methods. Part A. Methods and Devices. Academic Press, New York, 1: 1-515.

12. Zwillinger D (1989) Handbook of Differential Equations. Academic Press, Boston, 673 p.

13. Afonin SM (2015) Structural-parametric model and transfer functions of electroelastic actuator for nano- and microdisplacement. Piezoelectrics and Nanomaterials: Fundamentals, Developments and Applications. Ed Parinov IA Nova Science, New York, 9: 225-242.

14. Afonin SM (2017) A structural-parametric model of electroelastic actuator for nano- and microdisplacement of mechatronic system. Chapter 8 in Advances in nanotechnology. Bartul Z, Trenor J, Nova Science (Eds.), New York 19: 259-284.
15. Afonin SM (2012) Nano- and micro-scale piezomotors. Russian engineering research 32(7-8): 519-522

16. Afonin SM (2007) Elastic compliances and mechanical and adjusting characteristics of composite piezoelectric transducers," Mechanics of solids 42(1): 43-49.

17. Afonin SM (2014) Stability of strain control systems of nano-and microdisplacement piezotransducers. Mechanics of solids 49(2): 196207.

18. Afonin SM (2017) Structural-parametric model electromagnetoelastic actuator nanodisplacement for mechatronics. International Journal of Physics 5(1): 9-15.

19. Afonin SM (2017) Structural-parametric model of piezoactuator nanoand microdisplacement for nanoscience. AASCIT Journal of Nanoscience 3(3): 12-18.

20. Afonin SM (2016) Solution wave equation and parametric structural schematic diagrams of electromagnetoelastic actuators nano- and microdisplacement. International Journal of Mathematical Analysis and Applications 3(4): 31-38.

21. Afonin SM (2018) Structural-parametric model of electromagnetoelastic actuator for nanomechanics. Actuators 7(1): 1-9.

22. Afonin SM (2016) Structural-parametric models and transfer functions of electromagnetoelastic actuators nano- and microdisplacement for mechatronic systems. International Journal of Theoretical and Applied Mathematics 2(2): 52-59.

23. Afonin SM (2017) Parametric block diagrams of a multi-layer piezoelectric transducer of nano- and microdisplacements under transverse piezoelectric effect. Mechanics of Solids 52(1): 81-94.

24. Afonin SM (2018) Multilayer electromagnetoelastic actuator for robotics systems of nanotechnology, Proceedings of the 2018 IEEE Conference EIConRus, pp. 1698-1701.

25. Afonin SM (2009) Static and dynamic characteristics of a multi-layer electroelastic solid. Mechanics of Solids 44(6): 935-950.

26. Afonin SM (2010) Static and dynamic characteristics of multilayered electromagnetoelastic transducer of nano- and micrometric movements. Journal of Computer and Systems Sciences International 49(1): 73-85.

27. Afonin SM (2018) Electromagnetoelastic nano- and microactuators for mechatronic systems. Russian Engineering Research 38(12): 938-944.

28. Afonin SM (2018) Structural-parametric model of electro elastic actuator for nanotechnology and biotechnology. Journal of Pharmacy and Pharmaceutics 5(1):8-12.

29. Afonin SM (2018) Electromagnetoelastic actuator for nanomechanics. Global Journal of Research in Engineering. A: Mechanical and Mechanics Engineering 18(2): 19-23.

30. Afonin SM (2019) Actuator for nano biomedical research. Biomedical Journal of Scientific and Technical Research 19(3): 14300-14302.

31. Afonin SM (2019) Absolute stability of control system with electro magneto elastic actuator for nanobiomedicine. Biomedical Journal of Scientific and Technical Research 21(4): 16027-16030.

32. Bhushan B (2004) Springer Handbook of Nanotechnology. Springer, Berlin, New York, USA, p. 1222. 